\title{
REPORTE DE UN CASO: FRACTURA EXPUESTA DEL MEDIOPIE POR ACCIDENTE DE TRÁNSITO EN PACIENTE VIH/SIDA
}

\author{
CASE REPORT: EXPOSED MIDFOOT FRACTURE DUE TO TRAFFIC ACCIDENT IN HIV/AIDS PATIENT

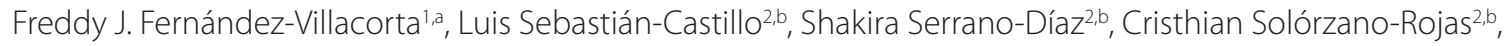

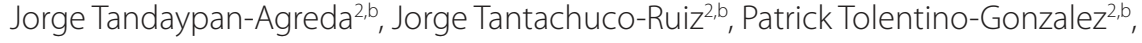 \\ Tony Chávez-Uceda², Esmeralda Torres-Soto $2, b$
}

\section{RESUMEN}

Actualmente hay una alta incidencia de accidentes de tránsito en el mundo, muchos de ellos provocan una discapacidad grave en las personas. Estas lesiones en las extremidades provocan alta morbilidad llegando incluso a una amputación. Esto se agrava en pacientes VIH/SIDA, sobre todo en el tratamiento y la evolución. Se presenta un caso clínico de un paciente con lesión severa del mediopie que termina en amputación y se hace una revisión del manejo quirúrgico del paciente traumatológico con VIH/SIDA.

Palabras clave: Fractura expuesta; Accidente de tránsito; VIH/SIDA (fuente: DeCS BIREME).

\begin{abstract}
Currently there is a high incidence of traffic accidents in the world, many of them cause severe disability in people. These limb injuries cause high morbidity even reaching an amputation. This is aggravated in HIV/ AIDS patients, especially in treatment and evolution. A clinical case of a patient with severe midfoot injury that ends in amputation is presented and a surgical management review of the trauma patient with HIV/ AIDS is made.
\end{abstract}

Key words: Exposed fracture; Traffic accident; HIV/AIDS (source: MeSH NLM).

\section{INTRODUCCIÓN}

Cada año mueren aproximadamente 1,35 millones de personas por accidentes de tránsito; de estos, entre 20 y 50 millones sufren traumatismos no mortales con una discapacidad permanente ${ }^{(1)}$. En el Perú las cifras de heridos por accidentes de tránsito durante el año 2018 fueron de 61512 y 32449 fallecidos ${ }^{(2)}$.

Las lesiones de la región media del pie comprenden aproximadamente un $5 \%$ de los traumatismos de pie. La mayoría de estas son lesiones son por traumatismos de alta energía. Por lo general, las lesiones en el mediopié implican fracturas múltiples o luxo-fracturas. Hasta el $30 \%$ de las lesiones en el mediopié se tratan de manera tardía, teniendo peores resultados ${ }^{(3)}$.

Desde 1983 al 2019 se han notificado un total de 120389 casos de infección por VIH en países en vías de desarrollo, de los cuales 43072 se encuentran

\footnotetext{
${ }^{1}$ Hospital Regional Docente de Trujillo, Trujillo-Perú.

${ }^{2}$ Universidad Nacional de Trujillo - Facultad de Medicina, Trujillo-Perú.

a Médico Traumatólogo.

${ }^{\mathrm{b}}$ Estudiante de Medicina.

Citar como: Freddy J. Fernández-Villacorta, Luis Sebastián-Castillo, Shakira Serrano-Díaz, Cristhian Solórzano-Rojas, Jorge Tandaypan-Agreda, Jorge Tantachuco-Ruiz, Patrick Tolentino-Gonzalez, Tony Chávez-Uceda, Esmeralda Torres-Soto. Reporte de un caso: fractura expuesta del mediopie por accidente de tránsito en paciente VIH/SIDA. Rev. Fac. Med. Hum. Abril 2020; 20(2):322-327. DOI 10.25176/RFMH.v20i2.2928 
en estado SIDA. La relación de VIH positivos es de 3,7 hombres a una mujer y en fase SIDA de 4,5 hombres a una mujer ${ }^{(4)}$. En varones, $55 \%$ de los casos se diagnosticaron entre los 20 a 34 años y en mujeres el $65 \%$ de los casos se diagnosticaron entre los 20 a 39 años ${ }^{(5)}$.

A nivel nacional en el 2018, Lima y Loreto son los departamentos con más casos de VIH/SIDA. En Lima se han reportado 2211 casos de VIH positivo y 861 en estadio SIDA, y en Loreto 411 casos de $\mathrm{VIH}$ y 23 en estadio SIDA. En el departamento de La Libertad 484 casos de $\mathrm{VIH}$ y 40 en estadio SIDA ${ }^{(6)}$.

En las especialidades quirúrgicas, el servicio de cirugía general presenta un mayor número de exposiciones a $\mathrm{VIH}(17,5 \%)$, seguido de traumatología (14,6\%). medicina interna presenta el $15 \%{ }^{(7)}$.

El impacto de la enfermedad en pacientes traumatológicos con una intervención quirúrgica aún no se ha aclarado por completo ${ }^{(8)}$. Existe evidencia contradictoria que el estado de $\mathrm{VIH}$, pueda incluir o no un recuento de CD4 comprometido, este aumente la probabilidad de infección u otros eventos adversos perioperatorios en cirugías electivas ${ }^{(9)}$. La preocupación puede ser aún mayor para pacientes en emergencia dada la naturaleza potencialmente inestable que se presentan por el trauma sufrido ${ }^{(10)}$.

Teniendo en cuenta lo anterior, el presente caso clínico tiene como finalidad exponer el abordaje terapéutico de la fractura expuesta de pie por aplastamiento en paciente VIH/SIDA, mostrar el tratamiento brindado y describir medidas de bioseguridad a usar en el manejo de un paciente traumatológico con VIH/SIDA.

\section{PRESENTACION DEL CASO}

Paciente masculino de 18 años de edad, natural y procedente de Ciudad de Dios (Pacasmayo), el cual tiene antecedente de diagnóstico por $\mathrm{VIH}$ sin tratamiento antiretroviral (TARV) en el hospital de Guadalupe dos meses antes. Mientras esperaba pasajeros en el paradero fue empujado por compañero contra un tráiler en movimiento, que transportaba piedras, pasándole la llanta trasera por el pie izquierdo. Presentó dolor, edema, exposición óseo tendinosa y herida con sangrado activo, por lo que fue trasladado a una posta médica para primeros auxilios y posteriormente referido por emergencia al hospital Regional Docente de Trujillo (HRDT) para mejor manejo definitivo.

Tres horas después del accidente arribó al HRDT despierto y con signos vitales estables. Se evidenció solución de continuidad de aproximadamente 10×10 cm con exposición de tendones, músculos y huesos del tarso y metatarso del pie izquierdo, dolor de moderada intensidad, movilidad del IV y $\mathrm{V}$ dedo del pie, disminución del pulso tibial posterior y pulso pedio no palpable; también presentaba una herida profunda en cara medial y lateral de rodilla izquierda. Se instauró tratamiento antibiótico triple: cefazolina, gentamicina y penicilina $\mathrm{G}$ sódica.

Se solicitó radiografías simples del pie izquierdo en dos proyecciones, encontrándose luxofractura de escafoides y de $1^{\circ}$ y $2^{\circ}$ cuña (Figura 1).

Se realizaron dos limpiezas quirúrgicas bajo anestesia, y posteriormente amputación desde el medio pie por el área de necrosis (figura 2). En el preoperatorio, el paciente se encontraba hemodinámicamente estable con una anemia leve (hemoglobina $9 \mathrm{gr} / \mathrm{dl}$, plaquetas $266000 \times \mathrm{mm} 3$, hematocrito 27,7\%, creatinina 0,7 mg/ dl, LDH 577 UI). Para el momento quirúrgico fueron aplicadas todas medidas de bioseguridad debido al alto riesgo de infección por $\mathrm{VIH}$ : uso de material descartable, guantes estériles, lentes, ropa descartable y campos estériles descartables. En la primera cirugía, en la zona afectada del pie izquierdo, una herida con cuerpos extraños (tierra, piedras), se procedió a una limpieza con 10 frascos cloruro de sodio al 0,9 \% y yodo povidona espuma al 1\%. Luego se procedió a fijación percutánea con 2 agujas Kishner de los huesos cuneiformes, desde el antepié al retropié (Figura 3). En el postoperatorio, se le administró tratamiento con vacomicina y meropenem.

Después de 2 días, se procedió a una segunda limpieza quirúrgica, evidenciándose diversas áreas de necrosis (Figura 4) por lo que se realizó debridamiento, pérdida de piel, tejido celular subcutáneo y músculo. Ya no presentaba mal olor. Después de 6 días de limpieza, se procede a amputación del mediopie y antepie, donde se encontraba área de necrosis (Figura 5). No hubo complicaciones durante la cirugía. El paciente fue intervenido dos veces para remodelación de muñón, luego de 15 días se observa herida abierta de 15x 10 $\mathrm{cm}$ con tejido de granulación en muñón de retropié. Continúa con curaciones diarias. 


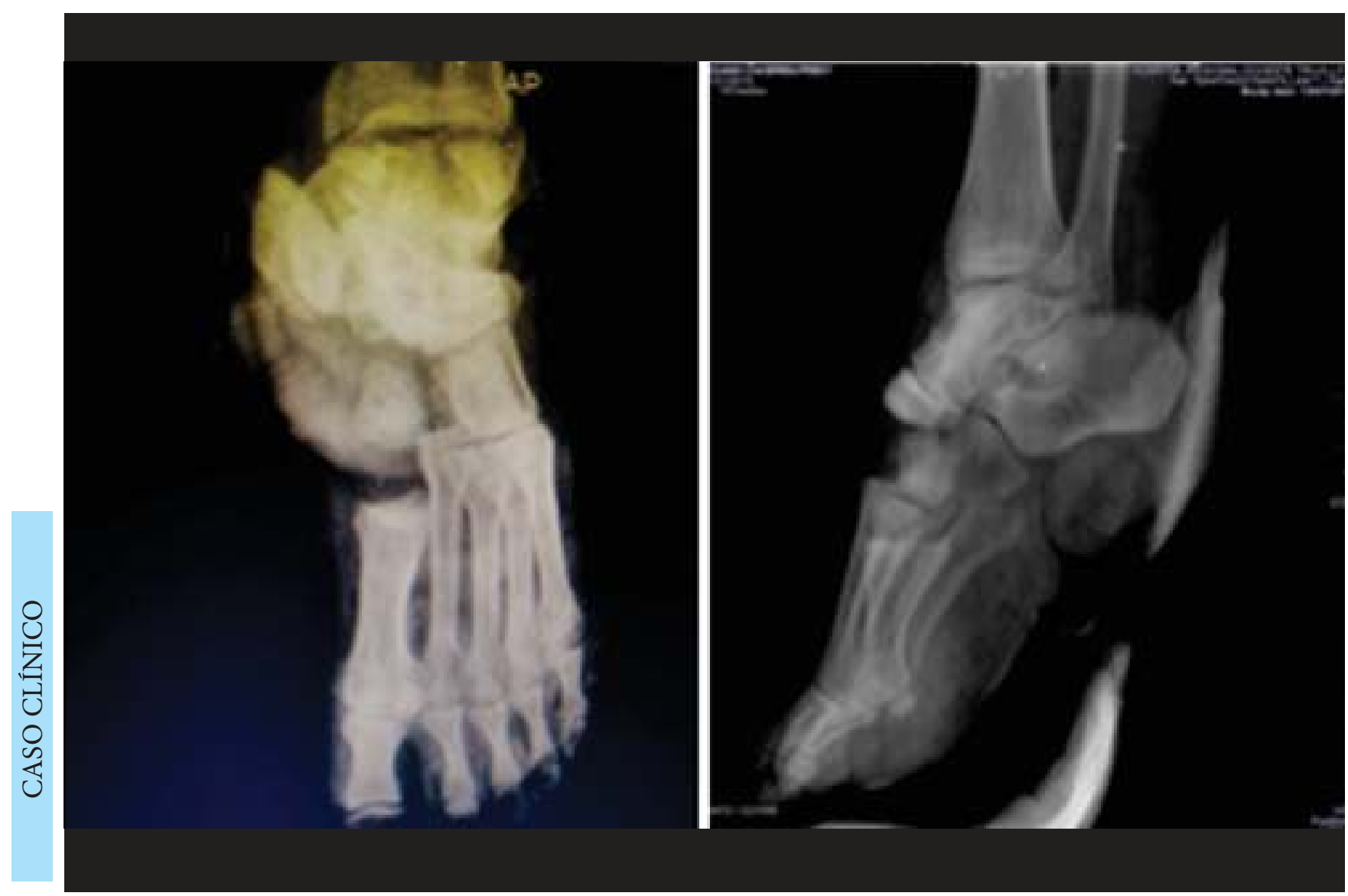

Figura 1. Radiografías del pie izquierdo en posición anteroposterior y lateral.

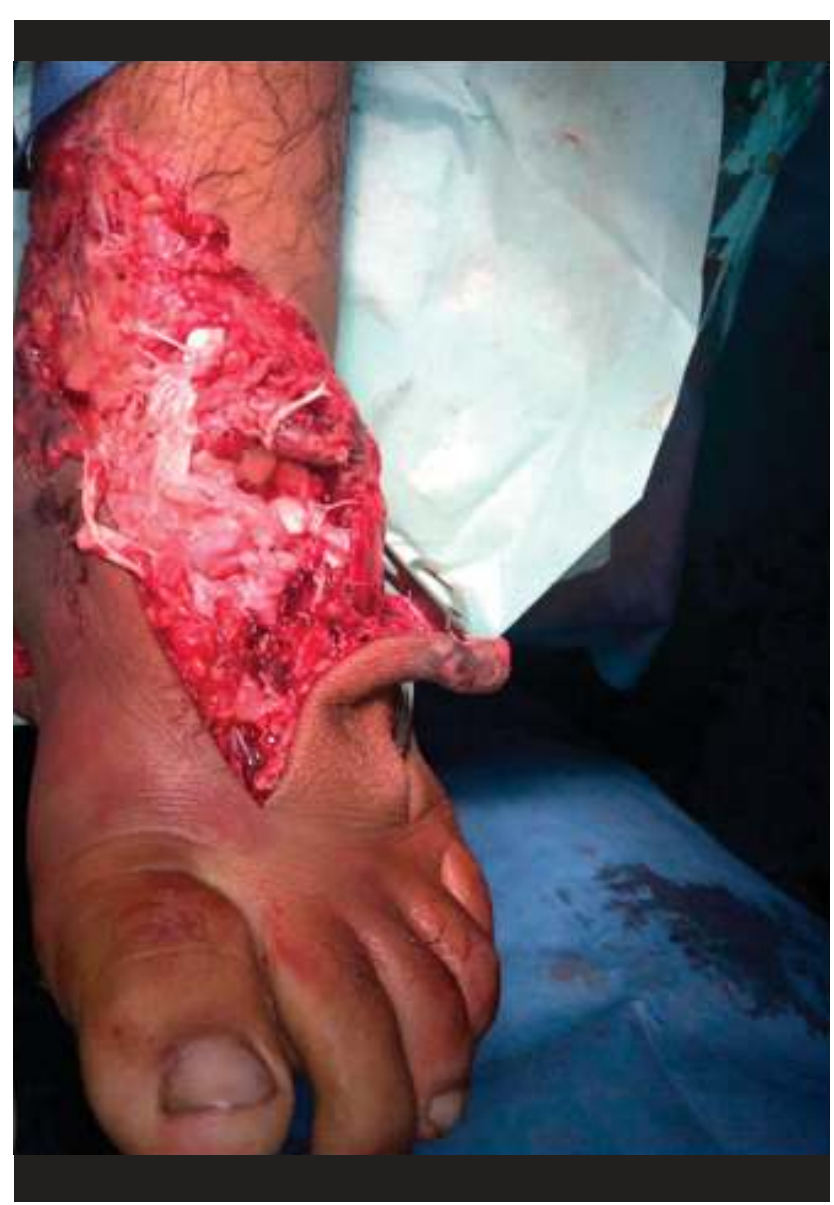

Figura 2. Primera limpieza quirúrgica.

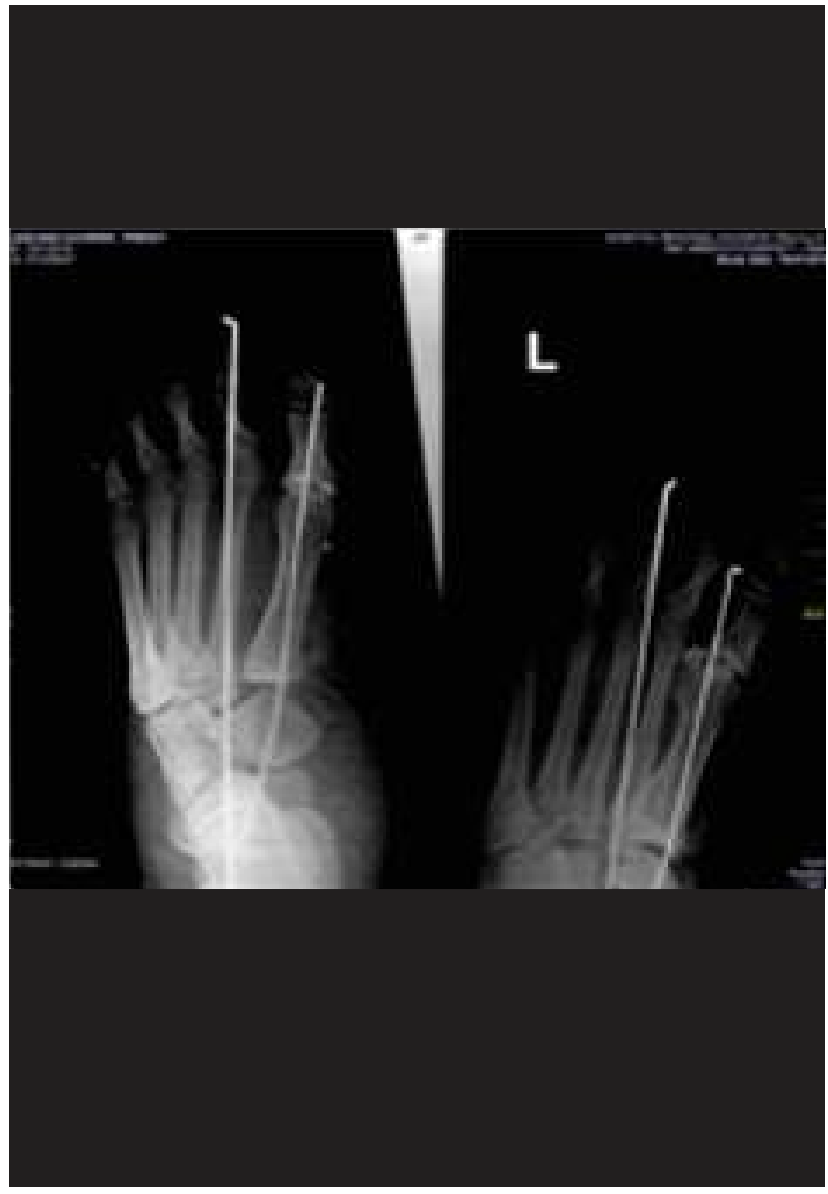

Figura 3. Fijación percutánea con agujas Kishner. 


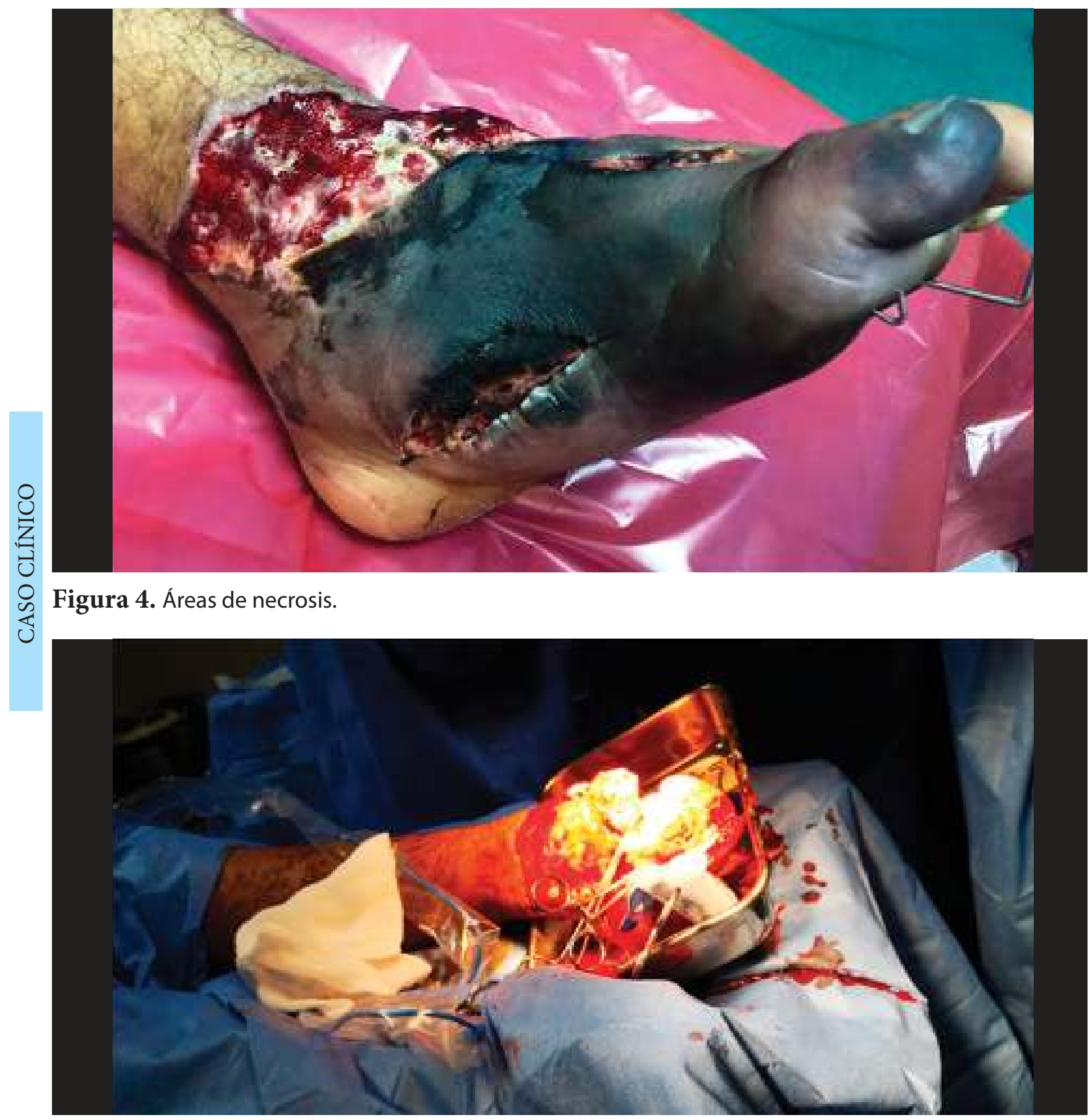

Figura 5. Muñón post-amputación.

\section{DISCUSIÓN}

En el ATLS (Advanced Trauma Life Support) ${ }^{(10)}$ se detalla una serie de estrategias para tratar lesiones musculo-esqueléticas. En el caso de fracturas abiertas y lesiones articulares abiertas, existe un gran problema, la contaminación bacteriana, hace que sean propensas a infección, de difícil curación y secuelas en la función. Por lo que, menciona, se debe eliminar las partículas de la herida lo antes posible, cubrirla con un apósito estéril húmedo; aplicar la inmovilización adecuada después y determinar cualquier afectación asociada de tejidos blandos, circulatorios y neurológicos. Se les debe tratar lo antes posible con antibióticos por vía intravenosa utilizando dosis máxima, las cefalosporinas de primera generación son de preferencia ${ }^{(11)}$. El retraso de la administración de antibióticos más allá de las tres horas está relacionado con un mayor riesgo de infección ${ }^{(11)}$. En el paciente se inició tratamiento antimicrobiano endovenoso con cefazolina, gentamicina y penicilina G sódica VEV durante 8 días, nunca hizo fiebre, sin embargo se presentaba dolor, rubor, tumefacción de tejidos blandos, mal olor, que podría complicarse a posibles piomositis, gangrenas, osteomielitis, entre otros, con el transcurso del tiempo, pensando en gérmenes más frecuentes como Staphylococcus aureus y Clostridium perfringes, entre otros, por lo que se le 
hizo un examen de cultivo más antibiograma.

En la clasificación de Gustilo y Anderson, el paciente presenta un grado IIIC debido a la herida contaminada y extensa, con daño vascular y nervioso ${ }^{(13)}$. Este grado de fractura expuesta presenta un mal pronóstico para el paciente. El puntaje MESS (Mangled Extremity Severity Score) estima la viabilidad de una extremidad después del trauma, para determinar la necesidad de rescate frente a la amputación. Este puntaje se obtiene a partir de la suma de puntos obtenidos en 4 parámetros que son: lesión de huesos y tejidos blandos, isquemia de extremidad, estado hemodinámico y edad $^{(14)}$. En este caso, se trata de un paciente de 18 años de edad, una presión sistólica sobre $90 \mathrm{mmHg}$, sin pulso pedio, con sensibilidad en la zona de lesión, y trauma de alta energía por lo que su puntaje total fue de 6 puntos. La escala sugiere que un puntaje mayor o igual a 7 es predictivo de amputación. Sin embargo, muchos autores cuestionan el uso del puntaje MESS porque no reemplaza la experiencia del cirujano. De hecho, Langer y Bosse determinaron que el MESS tenía baja sensibilidad (46\%). En cuanto a la amputación precoz esta cobra relevancia debido a que realizarla tempranamente reduce el riesgo de complicaciones posteriores a la intervención, el tiempo de recuperación y adaptación del paciente y mejoría en la calidad de vida del mismo.

En el paciente se realizó una desarticulación de Chopart con conservación de la almohadilla del talón para tratar el área de necrosis del mediopié y antepié. Además, se buscó el mejor pronóstico funcional posible, siendo esta amputación de elección desde el punto de vista de la rehabilitación al permitir la descarga de peso distal, lo cual es sumamente ventajoso a la hora de la confección protésica y hace que la carga de peso en la extremidad remanente durante la marcha sea mucho mejor tolerada que en el nivel transtibial. El inconveniente de esta amputación es que presenta un extremo distal voluminoso, lo cual puede ser complicado a nivel cosmético, pero las ventajas funcionales sobrepasan los impedimentos estéticos ${ }^{(15)}$.

En cuanto a la bioseguridad, toda exposición a fluido corporal contaminado conlleva un riesgo de transmisión del $\mathrm{VIH}$, aumentando en las siguientes circunstancias: si la penetración la lesión por pinchazo ocurrió con una aguja hueca en lugar de una sólida, si fue una penetración profunda de los tejidos blandos, si fue visible sangre en la aguja, si el paciente se encuentra en la etapa temprana de viremia o el paciente tiene SIDA avanzado (ambos tienen altos niveles de cargas virales) y en caso de exposición prolongada (sangre dentro de un guante) $)^{(15)}$. El uso de dos pares de guantes reduce el riesgo de contaminación por fluidos corporales, ya que el $98 \%$ de la sangre podría ser removida y eso también compensará defectos en el material de los guantes. Técnicas simples para reducir el riesgo ocupacional incluyen: corte con electrocauterio, evitar el paso de objetos punzantes mano a mano y el dogma de que las manos nunca deberían ser utilizado como retractores de tejidos.

Por lo tanto, el paciente con una fractura expuesta IIIC según Gustillo y Anderson producto de una lesión por aplastamiento. Con un compromiso severo de una o más extremidades no solo se debe abordar pensando en salvar la vida, sino en el individuo y su proceso futuro de reinserción familiar, social y laboral; se decidió realizar la amputación. Actualmente el tratamiento de pacientes con infección con VIH consume más recursos, además de peligros de contagio. Sin embargo, si la situación lo exige el cirujano debe tomar riesgos para mejorar el bienestar de otros como el caso presentado, siguiendo las medidas de bioseguridad universales y específicas. Por lo tanto, el cirujano debe actuar pensando en el pronóstico funcional, en base a la evidencia actual, logrando mejores resultados en todos los ámbitos de la vida de la persona a la que estamos tratando.

Contribuciones de autoría: Los autores participaron en la génesis de la idea, diseño de proyecto, recolección e interpretación de datos, análisis de resultados y preparación del manuscrito del presente trabajo de investigación.

Financiamiento: Autofinanciado.

Conflicto de interés: Los autores declaran no tener conflicto de interés en la publicación de este artículo.

Recibido: 06 de enero 2020

Aprobado: 28 de febrero 2020

Correspondencia: Tony Chavez Uceda. Dirección: Jr. Salaverry 555, Trujillo-Perú. Teléfono: 947366243

Correo:tony.comuc@gmail.com 


\section{REFERENCIAS BIBLIOGRÁFICAS}

1. WHO. Accidentes de tránsito [Internet]. Who.int. 2019 [cited 25 July 2019] Available from: https://www.who.int/es/news-room/fact-sheets/detail/roadtraffic-injuries

2. Centro Nacional de Epidemiología, prevención y control de enfermedades -MINSA. Situación de las lesiones causadas por accidentes de tránsito en el Perú. Boletín mensual; 2019.

3. Richter M, Wippermann B, Krettek C, et al. Fractures and fracture dislocation of the midfoot: occurrence, causes and long-term results. Foot Ankle Int 2001; 22:392.

4. Harries A, Suthar A, Takarinda K, Tweya H, Kyaw N et al. Ending the HIV/ AIDS epidemic in low- and middle-income countries by 2030: is it possible? F1000Res.2016;5:2328.

5. World Health Organization WH. Global HIV/AIDS response: epidemic update and health sector progress towards Universal Access. Geneva, Switzerland: World Health Organization; 2011.

6. Centro Nacional de Epidemiología, prevención y control de enfermedades -MINSA. Situación epidemiológica del VIH-SIDA en el Perú. Boletín mensual; 2018.

7. Kigera J, Straetemans M, Vuhaka S, Nagel I, Naddumba E et al. Is there an increased risk of post-operative surgical site infection after orthopaedic

surgery in HIV patients? A systematic review and meta-analysis. PLoS

One.2012;7: e42254.
8. Cacala S, Mafana E, Thomson S, Smith A. Prevalence of HIV status and CD4 counts in a surgical cohort: their relationship to clinical outcome. Ann R Coll Surg Engl. 2006;88:46-51.

9. Duane T, Sekel S, Wolfe L, Malhotra A, Aboutanos M et al. Does HIV infection influence outcomes after trauma? J Trauma. 2008;65:63-65.

10. Stewart R, Rotondo M, Hnery S, Drago M. ATLS Advanced Trauma Life Support. Student Course Manual 10 ed. American College of Surgeons [online] 2018 [consultado el 21 de Agosto del 2019]; 202-220

11. Burns K., Cone D., Portereiko J. Extracción compleja de la víctima y lesiones por aplastamiento. Prehospital Emergency Care. 2010; 4 (2): 240 - 4

12. Minisiterio de Sanidad, Servicios Sociales e Igualdad. Boletín S.U.E. 061 CEUTA dirección territorial de CEUTA atención primaria. España. 2013; 9 (46).

13. Elniel A, Giannoudis P. Open fractures of the lower extremity. EFORT Open Reviews. 2018;3(5):316-325.

14. Loja M, Sammann A, DuBose J, Li C, Liu Y, Savage S, et al. J Trauma Cuidado agudo Surg. 2017 mar; 82 (3): 518-523.

15. Espinoza M, García D. Niveles de amputación en extremidades inferiores: repercusión en el futuro del paciente. Revista médica clínica Condes. 2014; 25(2): 276-280.

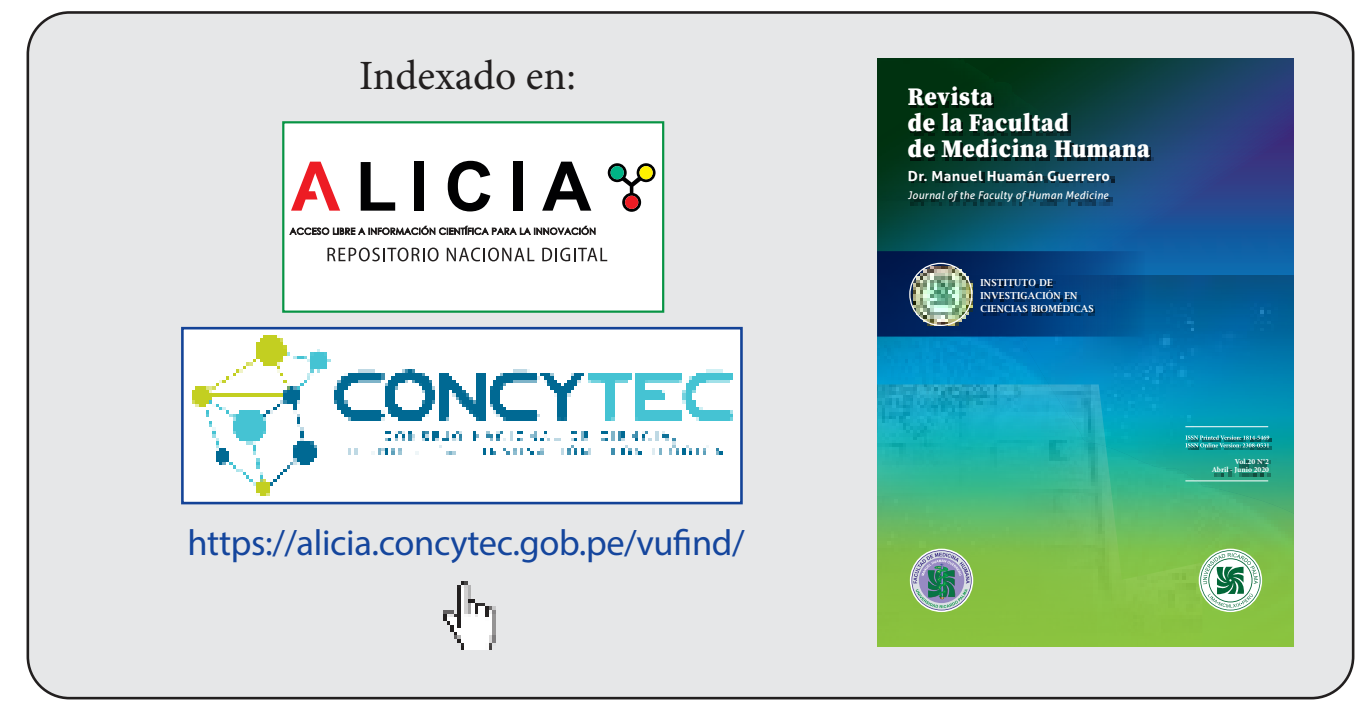

